\title{
DERRIERE LES CASES, LA CLASSE ou Comment initier les futurs professeurs des écoles à la BD ?
}

BEHIND THE CAPTIONS, THE CLASS or How introducing comics in teachers training?

\section{Sylvie Dardaillon et Christophe Meunier}

\section{(2) OpenEdition}

\section{Journals}

Édition électronique

URL : http://journals.openedition.org/trema/4853

DOI : $10.4000 /$ trema.4853

ISSN : 2107-0997

Éditeur

Faculté d'Éducation de l'université de Montpellier

Édition imprimée

ISBN : 979-10-96627-07-3

ISSN : 1167-315X

\section{Référence électronique}

Sylvie Dardaillon et Christophe Meunier, «DERRIERE LES CASES, LA CLASSE ou Comment initier les

futurs professeurs des écoles à la BD ? », Tréma [En ligne], 51 | 2019, mis en ligne le 01 avril 2019, consulté le 06 août 2019. URL : http://journals.openedition.org/trema/4853 ; DOI : 10.4000/

trema.4853

Ce document a été généré automatiquement le 6 août 2019

Trema 


\title{
DERRIERE LES CASES, LA CLASSE ou Comment initier les futurs professeurs des écoles à la BD ?
}

\author{
BEHIND THE CAPTIONS, THE CLASS or How introducing comics in teachers \\ training?
}

Sylvie Dardaillon et Christophe Meunier

1 Interrogés sur leurs pratiques scolaires, les enseignants évoquent leur rapport problématique aux littératures iconographiques, surtout la bande dessinée, qu'ils considèrent comme des supports difficiles d'accès, et qui laissent nombre d'entre eux démunis par manque de référence, de stratégies didactiques, de formation initiale (Dardaillon, 2009). Problèmes d'enseignants ou problèmes de lecteur? En effet, avant même d'envisager la didactisation de l'objet, encore faut-il le connaître, le pratiquer...

Une enquête en cours auprès d'un groupe d'étudiants inscrits en première année du master Métiers de l'Enseignement et de la Formation (MEEF) premier degré à l'ESPE Centre Val de Loire met en évidence la méconnaissance par la plupart d'entre eux du médium bande dessinée. La majorité des étudiants interrogés avoue être des lecteurs peu, voire pas, familiers de bandes dessinées. D'autres ont une approche erratique de cet iconotexte ${ }^{1}$ spécifique.

Comment alors faire prendre conscience aux étudiants-stagiaires que la bande-dessinée constitue un langage spécifique à travailler pour ses ressources propres, et définir les principales caractéristiques de ce langage? Comment utiliser ce langage, par exemple, pour conceptualiser en histoire géographie et en littérature grâce à des activités de lecture et/ou de production de bande dessinée? 


\section{La Bande dessinée, un médium méconnu des (jeunes) enseignants?}

Un recueil de représentations et de pratiques déclarées effectué de 2004 à 2007 sur la base d'un questionnaire et d'entretiens compréhensifs auprès d'une centaine de professeurs des écoles titulaires inscrits dans des stages de formation en didactique de la littérature, avait déjà permis de mettre en évidence une certaine gêne de ces enseignants vis à vis du médium BD (Dardaillon, 2009). Près d'un tiers des enseignants de cycle 3 interrogés, invités à noter les ouvrages lus dans leurs classes au cours des trois années précédentes, ne mentionnaient aucune BD. Seules quelques BD de facture classique comme Angelot $d u$ lac d'Yvan Pommaux, publié au milieu des années 1990, étaient citées comme ayant fait l'objet d'une lecture avec les élèves. Quelques enseignants évoquaient la lecture d'extraits ou déploraient l'absence de séries dans les écoles, leur préférence allant aux «BD à caractère historique (Alix, Vasco), de science-fiction (les deux séries de Léo) ou humoristiques (Lagaffe, Petit Spirou, Kid Paddle) ».

5 À travers leurs réponses, les enseignants interrogés ont donné à voir leurs représentations des élèves et de la littérature. Considérée comme amplement dominante dans la culture des élèves, la BD est décrite comme cristallisant le conflit entre lecture privée et lecture scolaire, puisqu'elle serait "facile », " rapide à lire », sans exigence de compétences particulières. Malgré son entrée dans les programmes et sur la liste de référence dès 2002, ce genre iconotextuel reste donc, selon les termes des enseignants interrogés, « entaché de loisir et de facilité ", l'école devant s'appliquer à en détourner l'enfant pour l'amener ailleurs, vers d'autres genres littéraires.

6 Présentant parfois le coût des albums comme un obstacle à leur présence dans les classes, la plupart des enseignants qui s'exprimaient dans cette première étude évitaient de se questionner sur les raisons plus profondes de leur non choix. On notait parfois même un rien de condescendance dans les propos: "Je n'ai aucune série (mais je ne fais aucun effort pour en trouver...) ». Enfin, album et bande dessinée étaient évoqués de manière problématique, certains avouant ne pas savoir «comment prendre la substantifique moelle», se voyant travailler sur les codes mais pas sur le sens, sur une planche isolée mais pas sur un récit complet.

7 Afin d'observer si une quinzaine d'années après l'entrée de la BD dans les programmes de l'enseignement primaire, et à une génération de distance, le rapport à la BD avait changé chez les enseignants, une nouvelle interrogation a été réalisée en mars 2018 auprès d'un groupe de vingt-huit étudiants de Master MEEF que nous préparons à passer le concours de recrutement au professorat des écoles. Ce groupe est essentiellement féminin (86\%), à l'image de ce Master. Il est constitué d'étudiants sortant en grande majorité d'une licence (22 cas sur 28) mais également de quatre étudiants issus d'un autre master et d'un étudiant détenteur d'un diplôme d'éducateur. Autre élément à souligner ici : aucun des étudiants de ce groupe ne venait d'une UFR de Lettres. Comme le montre le graphique 1, les étudiants, pour la plupart, viennent des sciences humaines (Psychologie, Sociologie, Histoire, Géographie, Sciences de l'Education et du Langage). 
Tableau 1 - Origine disciplinaire des étudiants interrogés $(n=28)$

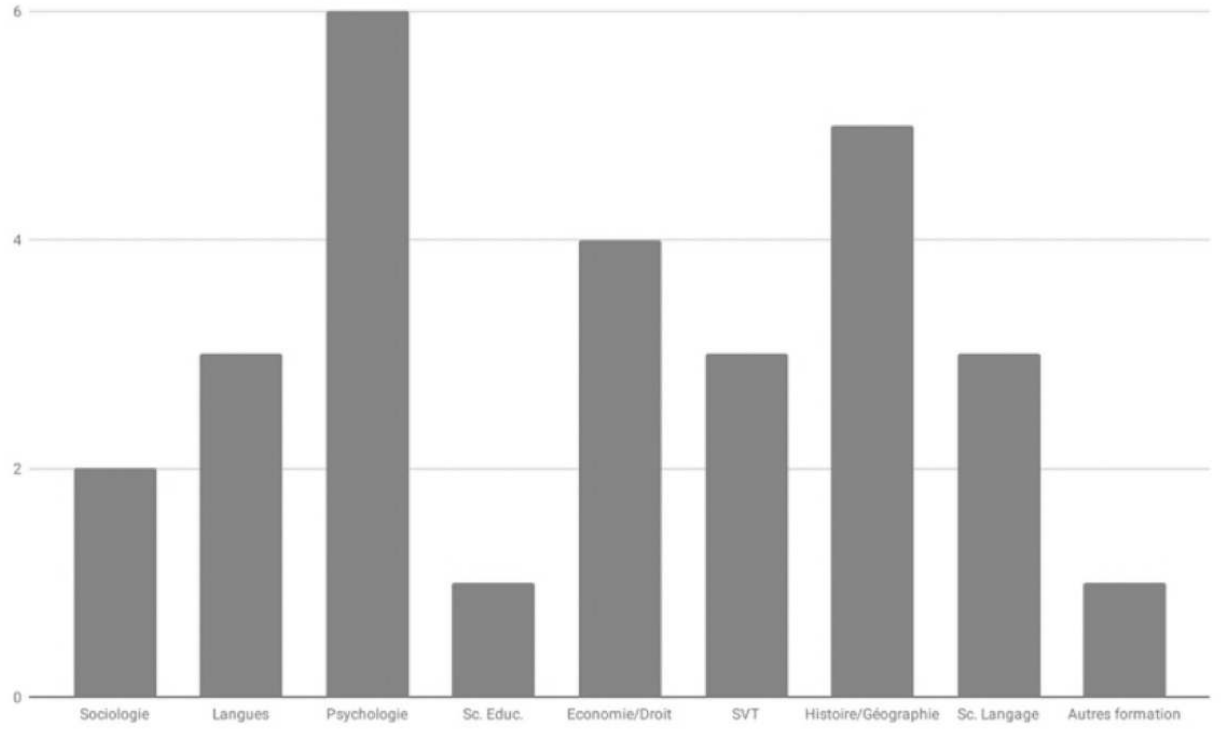

8 La moyenne d'âge du groupe est de 25 ans et la grande majorité (cf. tableau 2) des étudiants qui le constitue sont des jeunes nés entre 1991 et 1997. Cette observation aura son importance quand il s'agira d'analyser leurs références de lecture bédéistique durant leur enfance. La majorité du groupe correspond en effet à la génération qui a grandi avec le phénomène Titeuf dont le premier album sort en 2001 et qui était destiné aux enfants de 8/10 ans (âge que la majorité du groupe avait alors) ainsi qu'au phénomène Lou, personnage issu de la revue Tchô, et sorti en 2004, touchant plutôt un lectorat de jeunes filles de 11/12 ans.

Tableau 2 - Répartition des étudiants interrogés par âge

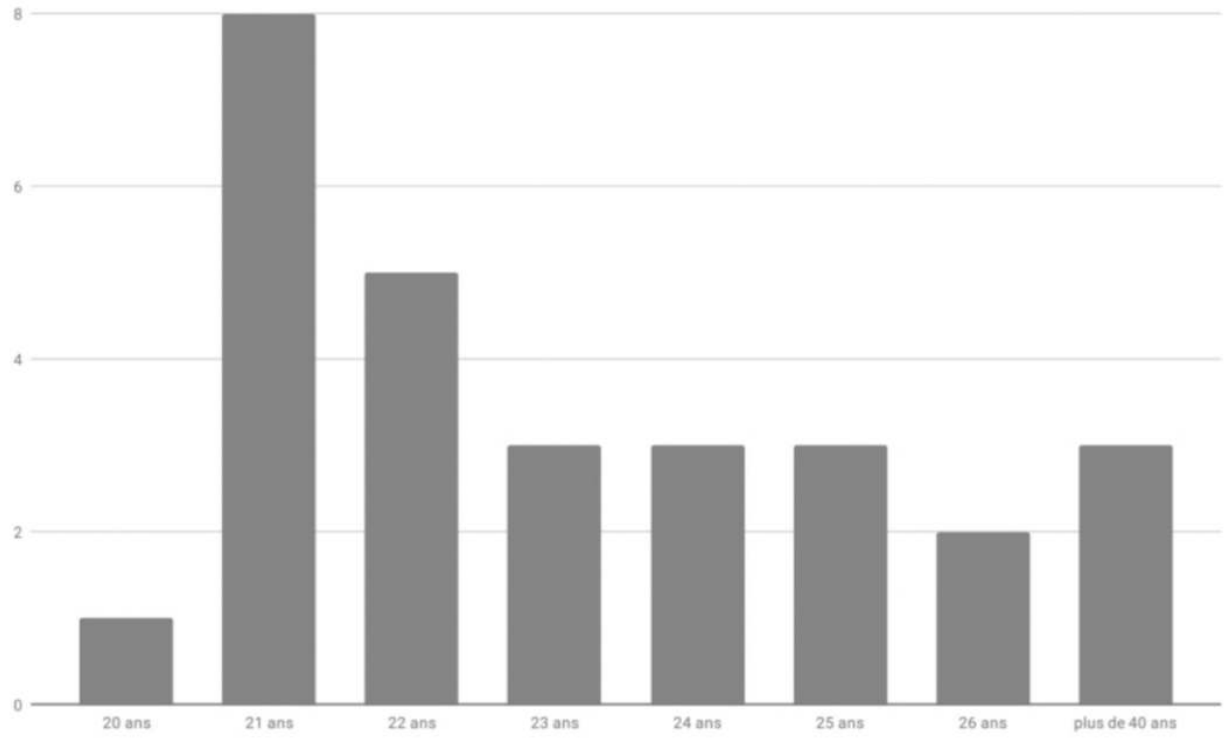

9 Un tiers des étudiants interrogés se déclarent avoir été lecteurs de BD dans l'enfance, la proportion accusant une forte baisse à l'âge adulte : sur ceux qui écrivent en avoir lu 
souvent dans l'enfance, la moitié n'en lit plus que de temps à autre, un tiers n'en lit plus jamais, par manque de temps ou d'intérêt. Parmi les lectures d'enfance, Tintin, Astérix, Titeuf, Tom-Tom et Nana sont fortement plébiscités (entre 11 et 19 occurrences sur 28) puis viennent Les Schtroumpfs et Spirou (6 occurrences) et plus rarement Lucky Luke, Mickey, ou encore des titres plus récents, ancrés dans les pratiques adolescentes comme Lou ou Les Nombrils. La plupart des étudiants semble par ailleurs avoir peu changé de corpus entre l'enfance et l'état de jeune adulte, néanmoins certains font référence à des auteurs s'adressant davantage à un lectorat adulte (Bilal, Moebius, Larcenet). On constate plus généralement un changement dans la manière dont les références sont convoquées : par séries pour les lectures d'enfance, par auteurs ou titres pour les lectures adultes. Au-delà des seules BD, les lectures d'enfance en générale, en grande partie attachées au contexte familial et plus rarement scolaire, sont manifestement fortement liées à l'affect tandis que les lectures adultes semblent liées au contexte universitaire conduisant les étudiants vers des ouvrages scientifiques, théoriques et faisant passer à l'arrière-plan les lectures plaisir/loisir, dont celles de BD. Si les étudiants interrogés y reviennent, c'est souvent par la médiation d'un conjoint ou d'enfants.

Le questionnaire que nous avons fait passer se concentrait également sur les stratégies de lecture mises en œuvre par les étudiants pour lire la bande dessinée. La grande majorité des étudiants n'a pas pu ou pas su répondre à cette question. Parmi les réponses exprimées, trois étudiants déclarent lire d'abord les images puis le texte, deux lisent image et texte dans un même mouvement, deux enfin disent pratiquer une première lecture d'ensemble puis une reprise des détails, laissant supposer par-là que le médium implique pour eux la relecture. Les étudiants interrogés soulignent par-là l'originalité du médium bande dessinée qui propose sur une même planche plusieurs vignettes et plusieurs blocs de texte disposés d'une manière particulière et logique, tous ces éléments entretenant des relations complexes d'articulation à la fois linéaires (à l'échelle de la séquence de vignettes) et translinéaires (à l'échelle de la planche ou multicadre) comme l'analyse Thierry Groensteen (1999).

11 Certains étudiants expriment clairement le fait que la bande dessinée possède des " codes ", un « langage propre » auxquels ils n'ont pas toujours été suffisamment initiés. La bande dessinée impose notamment une lecture « active » des planches à l'instar de ce que faisait remarquer Chris Ware en en 2008 dans le numéro 10 de la revue Humoresques :

Pour moi, la grande différence entre le cinéma et la bande dessinée est que le spectateur de cinéma est relativement passif, alors que le lecteur de BD s'investit beaucoup plus dans la construction même du sens. Si on devait faire une comparaison avec la musique, je dirais ceci : quand on regarde un film, c'est comme si on écoutait de la musique enregistrée, alors que quand on lit une BD, c'est comme si on lisait une partition. C'est à nous, lecteurs, de faire jaillir la musique de cette partition. (Ware, 2008)

En outre, la lecture de la bande dessinée n'est pas nécessairement linéaire. Avec l'appui des nouvelles technologies, quelques équipes de chercheurs ont mené des expériences de eye-tracking (ou oculométrie) pour tenter de comprendre les procédés de lecture de la bande dessinée et les différences avec les textes conventionnels. Les études de Nikagawa en 2004 et d'Allen et Ingulsrud en 2007 montrent qu'il n'existe pas de réel consensus entre ceux qui lisent d'abord les images puis les textes et ceux qui lisent d'abord les textes puis les images. Il semble que le regard porté sur une planche ne soit jamais prioritairement linéaire mais circulaire. Avant d'amorcer une lecture linéaire des strips, le lecteur d'une planche ou d'une double-planche de BD porte prioritairement, puis régulièrement au 
cours de sa lecture, un regard circulaire sur l'ensemble de la ou des planches qu'il a sous les yeux. La lecture tabulaire semble ainsi être concomitante de la lecture linéaire. Ces conclusions peuvent expliquer le désarroi de certains des étudiants interrogés, plus habitués à la lecture linéaire de romans.

La dernière partie du questionnaire invitait les étudiants à se projeter dans leur futur rôle d'enseignants. A la question «Pensez-vous intégrer la bande dessinée dans vos futurs cours? Pourquoi ?», 25 d'entre eux affirment envisager de proposer la lecture de BD à leurs élèves contre deux qui s'y refusent et un dernier qui déclare ne pas avoir tranché pour l'instant. Les justifications positives vont dans le sens de la découverte par les élèves de stratégies de lecture différentes (4 occurrences), de la découverte d'un genre nouveau (4 occurrences). D'aucuns mettent en avant l'intérêt d'un médium faisant interagir texte et image dont on travaillera l'analyse à cette occasion (2 occurrences), ils mettent également l'accent sur l'intérêt d'amener les élèves à utiliser le langage et les codes de la BD (2 occurrences). Pour d'autres, aborder la BD pourrait s'avérer facilitateur de l'entrée en lecture (3 occurrences), ils y voient un support « ludique ». Pour ces derniers, la BD "plait aux enfants", permet de pratiquer des jeux de lecture (4 occurrences), et suscite la créativité (3 occurrences). Pour d'autres enfin, la BD est « un bon support », qui présente «beaucoup de points forts» en tant que support interdisciplinaire permettant l'acquisition de connaissances et l'ouverture de débats, en Enseignement Moral et Civique notamment. Les questionnaires font toutefois apparaître quelques réserves: certains étudiants imaginent mal comment l'utiliser ou expliquent leur manque d'intérêt par une méconnaissance du domaine ou de l'outil. Les réponses font par ailleurs apparaître un flottement terminologique qui nécessiterait de redéfinir et/ou mettre en débat les termes tels que lecture, genre, outil et support.

L'enquête que nous avons réalisée ne fait que confirmer des impressions que nous avions déjà auprès de nos étudiants. Ces derniers ont une culture très mince de la bande dessinée, et, tout en considérant qu'il s'agit d'un médium "facile», ils présentent certaines difficultés à s'en emparer et ont subséquemment rarement l'idée qu'elle puisse constituer un support d'enseignement. Forts de ces résultats, nous avons donc décidé de consacrer un « temps fort » de la formation de la première année de MEEF premier degré à la découverte et à l'utilisation de la BD en classe.

Depuis la rentrée 2016, nous organisons à destination de tous les étudiants inscrits en première année du Master MEEF Premier Degré un temps fort d'une demi-journée autour de la bande dessinée à l'école. Cette opération débute par une conférence en plénière sur l'histoire de la bande dessinée et une présentation des caractéristiques du médium.

Cette première approche, à la fois dense et très générale, d'une heure suscite souvent beaucoup d'interrogations de la part des étudiants. Ils en apprécient énormément le contenu à en juger par l'évaluation réalisée en fin de temps fort. Cette conférence est suivie de plusieurs ateliers d'une heure-trente sur lesquels les étudiants se sont inscrits au préalable. Dans cet article, nous avons souhaité porter notre attention sur trois exercices réalisés lors de ces ateliers. Le premier est un exercice de lecture de planches qui se propose de travailler sur la réception du lecteur. Le deuxième exercice est un exercice de réduction de planches permettant de mettre en évidence les «codes » et les "modes» de lecture du médium BD. Enfin, le troisième exercice est un exercice «d'hybridation/fragmentation ${ }^{2}$ » qui se propose de montrer comment le langage de la bande dessinée permet de conceptualiser dans le cas particulier de l'histoire enseignée à l'École primaire. 


\section{Écrire un récit à partir d'une planche pour expliciter des stratégies de lecture}

17 Le premier exercice porte sur les pages 10 et 11 (soit la deuxième double-planche) du premier tome d'Angelot du Lac d'Yvan Pommaux (Bayard, 2010), BD particulièrement populaire dans les écoles en raison notamment d'une esthétique claire et efficace qui la rend facilement lisible tant pour les élèves que pour des adultes enseignants parfois peu familiers du médium. Ces planches permettent de travailler sur l'incipit dans le récit iconotextuel : contextualisation historique par la prise d'informations conjointes entre texte et image, perception des effets de dramatisation provoqués par les choix de montage et de cadrage, interprétation des rapports hiérarchiques entre les personnages qui font incursion sur la page, réflexion sur la valeur programmatique de cette double planche.

\section{Image 1 - Y. Pommaux, Angelot du Lac, p.10}
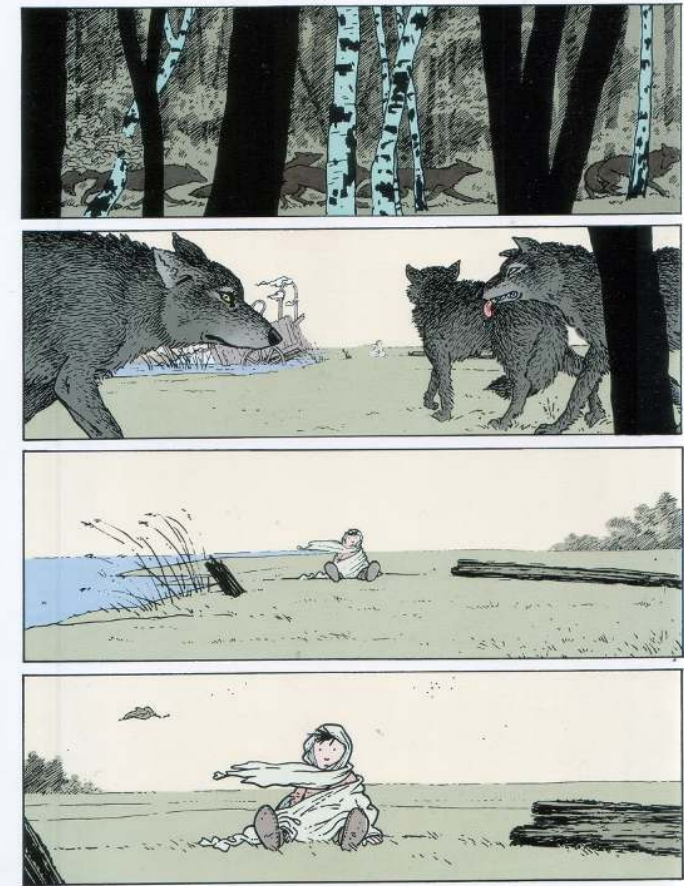

10 


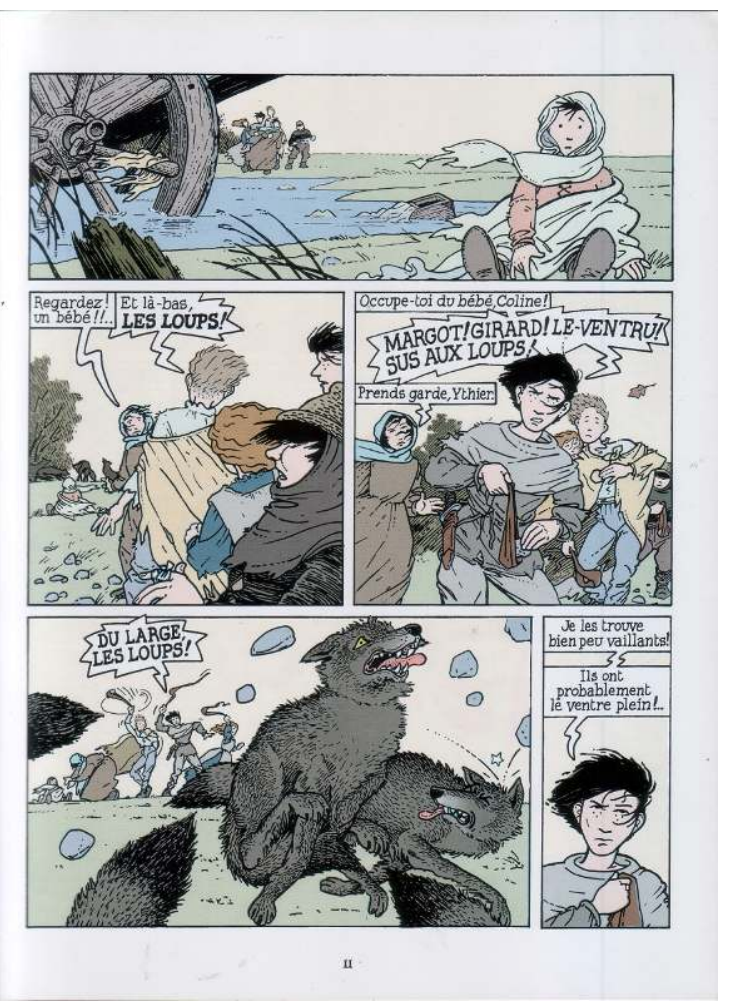

Avant de procéder à un débat interprétatif collectif, les étudiants sont invités à observer une reproduction de la double-planche et à traduire ce qu'ils en interprètent en un court récit d'une demi-page. Il s'agit de passer par le biais de l'écriture individuelle pour problématiser la lecture de planches : quels sont les éléments privilégiés par le lecteur, les détails ignorés, en quoi l'ordre proposé par le scripteur pour son récit romanesque coïncide-t-il ou non avec celui de la BD, comment prend-il en compte les divers plans de l'image? Quelques-uns de ces récits sont ensuite oralisés, comparés, discutés pour commenter les stratégies de lecture dont ils sont la manifestation et construire les éléments d'une interprétation collective basée sur la lecture de détail.

Les étudiants se prêtent volontiers au jeu de la narration à partir de l'incipit de Angelot $d u$ Lac qu'aucun ne semble connaître, produisant des récits de facture très diverse d'un point de vue discursif. Ainsi, l'un des récits, pour le moins lapidaire, se contente d'une simple prise en compte de l'événement: "Des hommes viennent sauver un enfant abandonné sur le point de se faire manger par des loups ». Il s'agit là, d'un certain point de vue, d'un détournement de la consigne qui rend compte du contenu narratif global, reconstitué $a$ posteriori sans prise en compte du mouvement de la double-planche, de l'inscription historique, des relations entre les personnages. La caractérisation de l'enfant comme « abandonné » montre toutefois une prise en compte implicite et immédiate d'éléments iconiques significatifs de l'isolement et de la solitude de l'enfant.

D'autre récits construisent à partir de la double page une amorce de schéma narratif avec situation initiale, complication, résolution et situation finale, sans péripéties toutefois, comme c'est le cas dans cette production :

Dans une forêt, une meute de loups affamés part en chasse d'une proie. À la lisière de cette forêt, un enfant assis dans l'herbe, ne bouge pas, ne pleure pas et ne semble 
pas apeuré. Un groupe de paysans passant par-là chasse les loups et récupère l'enfant. compte :

Dans une sombre forêt, une meute de loup flaire un gibier. Il ne s'agit pas d'un gibier comme les autres, c'est un enfant abandonné au milieu d'une prairie, au milieu des décombres d'une charrette. Les loups s'en approchent doucement. Au même moment, un groupe de personnes s'approche :

- Regardez, un bébé !! dit l'un

- Et là-bas les loups, dit un autre

- Occupe-toi du bébé Coline !

- Margot, Girard, le Ventru, sus aux loups ! dit celui qui semble être le chef

Avec leur fronde ils envoient des pierres et les loups prennent le large.

Ce récit prend en compte le rythme de la narration iconique, la mise en scène que propose l'effet de ralenti et de champ-contrechamp des premières vignettes étirées sur la bande puis l'accélération provoquée par le découpage de la bande en trois vignettes. L'étudiant semble avoir bien perçu la hiérarchie mise en évidence par la composition de l'image et les jeux de cadrage: effectivement, Ythier, le personnage qui apparaît en premier et gros plan est le meneur du petit groupe d'orphelin. Cet étudiant intègre par ailleurs la dimension sonore de la bande dessinée, les dialogues inscrits dans les bulles. Sur certains éléments, les interprétations du groupe divergent quant aux relations entre personnages : s'agit-il d'un groupe d'adolescents? de villageois ? existe-t-il un lien entre l'enfant abandonné et eux ? sont-ce des amis venus à la rescousse, inquiets du fait que la famille en déplacement ne soit pas encore arrivée à bon port? Les éléments contextuels sont par ailleurs peu investis même si l'un note qu'il s'agit d'une forêt de bouleaux, un autre imagine Angelot abandonné au bord de la mer, un autre l'appelle Pierre. Aucun étudiant ne fait le lien entre cette scène inaugurale et le titre de la série au point d'identifier ce petit «ange » abandonné près des roseaux à Angelot du Lac. De même, si certains ont bien repéré les frondes, repris les noms et expressions des personnages, aucun n'ancre explicitement son récit dans le Moyen Age.

La confrontation des récits ainsi que la reprise de la double-planche projetée permettent de revenir sur ces éléments perçus par le lecteur mais non intégrés à la narration, de revenir sur des stratégies de lecture hâtives qui vont directement au sens global sans prendre en compte les spécificités narratives du médium BD.

\section{Manipuler des planches pour saisir les spécificités de la narration en $B D$}

Le deuxième exercice est un exercice de manipulation qui s'inspire des travaux de l'OuBaPo. L'Ouvroir de Bande Dessinée Potentielle, se référant lui-même aux expériences littéraires de l'OuLiPo, est une association d'auteurs, de théoriciens et d'amateurs de bande dessinée, créée au sein de la maison d'édition L'Association en 1992. Les membres fondateurs, parmi lesquels on pouvait compter Thierry Groensteen, Jean-Christophe Menu, Etienne Lecroart et Lewis Trondheim, souhaitaient créer des bandes dessinées sous la contrainte artistique volontaire.

L'exercice de « réduction » est un grand classique des ateliers de l'OuBaPo. Il s'agit, pour le cas de l'atelier mis en place au sein du temps-fort, de demander à des binômes 
d'étudiants de réduire en une page un récit iconotextuel court développé sur deux pages. Le choix s'est porté sur un récit de Mongo Sise, "Matamata et Pilipili », paru dans le Journal de Spirou ${ }^{3}$. Le récit iconotextuel est court, commençant par une case d'introduction sur le premier strip de la première page et se terminant par une case de chute au dernier strip de la seconde page. Les deux pages sont liées par deux cases d'enjambement dans lesquelles d'une part la moto du gendarme puis l'auto des deux héros sortent de la case offrant la continuité d'une course-poursuite entre les deux véhicules. Le schéma de cette double-page est donc assez classique et n'offre pas de difficulté de lecture. De surcroît, cette planche propose un certain nombre de codes cartoonistiques (enjambement, case introductive, case de chute, onomatopées, case, bande, planche, bulle, cartouche) sur lesquels il est possible de revenir avec les étudiants dans un second temps. Comme matériel, les étudiants n'ont droit qu'aux ciseaux, à la colle et à une feuille A4 sur laquelle ils doivent sélectionner des cases sans les remanier et les disposer sans modifier le sens de la double-page.

À travers cet exercice, les étudiants, qui ne semblent pas avoir rencontré de difficultés majeures, ont pu manipuler un certain nombre de caractéristiques qui constituent la spécificité de ce médium. Le découpage et la sélection les ont conduits à travailler l'ellipse. L'agencement et le collage des vignettes sur une feuille A4, en respectant le sens du récit initial, les ont amenés à travailler la séquentialité et la mise en page, ou plus précisément ce que Groensteen nomme la «spatiotopie» (Groensteen, 1999). Les productions des étudiants peuvent être regroupées en deux catégories de planches. Afin de rendre compte des résultats obtenus, nous avons retenu deux exemples de planches que nous analyserons. Dans les deux cas, les auteurs ont conservé le titre en tête de page. Ils ont également conservé la construction de la planche en quatre strips.

Image 3 - Planches de type A et de type B réalisées par les étudiants interrogés

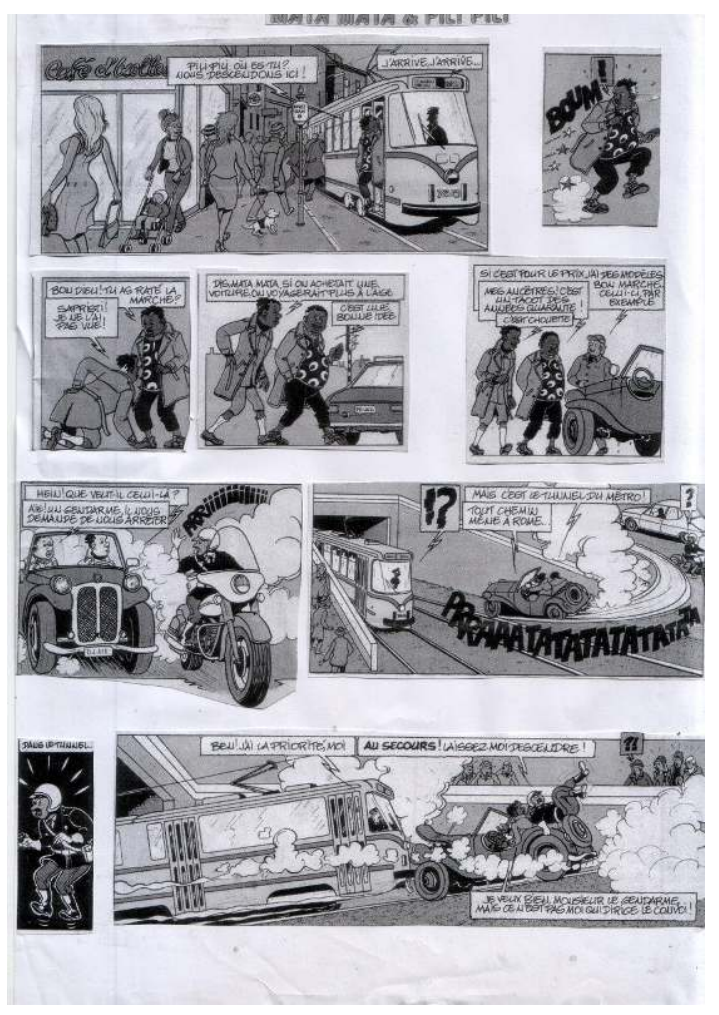




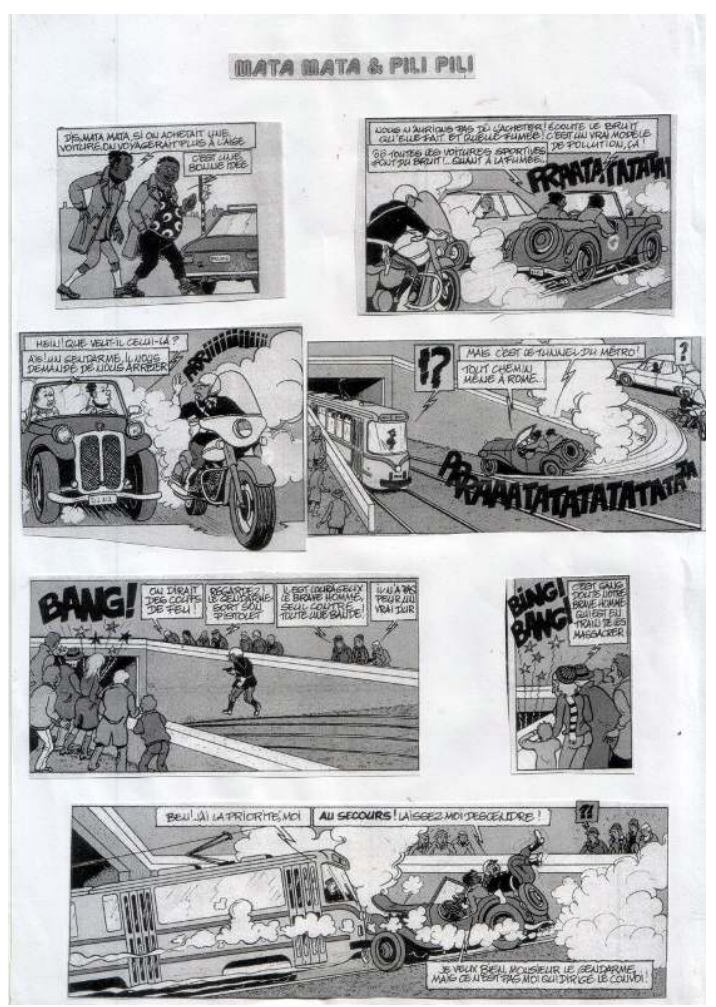

La planche de type A a conservé neuf cases sur les dix-neuf que compte l'ensemble de la double-page, alors que celle de type B n'en a conservé que sept. La planche de type A débute par la case d'introduction qui est une case longue de situation dans laquelle les personnages sont tous de pied, dans un décor présenté dans son ensemble. L'action est située dans l'espace. Cette première case de situation est une sorte de « règle d'or » posée par Jigé et Franquin, dessinateurs de Spirou, dans un livre d'entretiens menés par Philippe Vandooren en 1969. Les auteurs de la planche de type B ont fait le choix de débuter par la dernière case du deuxième strip de la planche originale. Ils écartent la possibilité de situer l'action dans l'espace mais davantage dans la narration: les deux amis congolais souhaitent acheter une voiture. L'achat de l'automobile est éclipsé et l'on passe, après un vide intericonique, à la poursuite par le motard de la gendarmerie. On rejoint alors la trame narrative de la planche de type A.

Les deux planches se terminent par la même case de chute qui prend toute la longueur d'un strip. Les auteurs de la planche de type $\mathrm{A}$ ont décidé d'inclure en première case $\mathrm{du}$ dernier strip une case noire très étroite montrant le motard dans le tunnel qui permet de faire une transition logique entre la case 2 du strip 3 et la case 2 du strip 4. Cette petite case noire qui montre le gendarme surpris dans le tunnel remplace le strip 3 de la planche de type B.

Ces analyses sont apportées oralement lors d'une phase de mutualisation autour des diverses planches affichées avec les étudiants. Ce moment permet à l'animateur de l'atelier d'identifier différentes caractéristiques qui participent du « langage de la bande dessinée ». Ainsi, nous pouvons insister sur trois éléments essentiels que l'exercice a mis en évidence. 

cases totales de la planche originale a permis de faire travailler les étudiants sur l'ellipse, ce que nous pouvons souligner à travers la comparaison de planches réalisées. Ensuite, il est important de faire remarquer aux étudiants que, quelle que soit la sélection de cases effectuée, l'ordre initial des cases est respecté. La linéarité de la lecture est conservée car, pour reprendre le titre d'un ouvrage de référence écrit par Will Eisner en 1985, la bande dessinée est «un art séquentiel». Enfin, nous pouvons remarquer que tous les auteurs des planches réalisées accordent une grande importance à la mise en espace, à la «spatiotopie» (Groensteen, 1999). Chaque planche respecte les quatre strips originaux, les vides intericoniques sont harmonieux et la composition de certains strips est soignée jouant avec l'intrigue et le suspens amenant la chute. termes de vocabulaire en lien avec le système de la bande dessinée à l'instar des mots suivants : case, strip, planche, onomatopée, bulle ou cartouche. Il a permis aux étudiants de manipuler une planche en la déconstruisant et en la reconstruisant tout en en conservant la narration et le sens.

\section{Produire des planches, faire dialoguer images et textes pour s'approprier des connaissances}

Le dernier exercice est un peu plus long dans sa réalisation et met en jeu savoirs historiques à acquérir à partir de documents sources datant de la Renaissance (extraits de Gargantua et de Pantagruel de François Rabelais, gravures du XVIe siècle) et compétences langagières (écrites et iconotextuelles). L'exercice se compose de trois phases successives. Dans un premier temps, les étudiants sont répartis en cinq groupes qui vont chacun travailler sur une thématique particulière qui permettrait de mettre en évidence les changements opérés entre le Moyen-âge et la Renaissance : l'éducation, la religion, les guerres, la vie quotidienne et les voyages. Ces changements seront abordés à partir de l'œuvre littéraire d'un auteur qui se situe sur la période charnière de la fin du Moyen-âge et du début de la Renaissance, François Rabelais.

Ainsi, chaque groupe découvre deux textes, l'un tiré de Gargantua et l'autre de Pantagruel, illustrant chacun des thèmes évoqués précédemment. Une première question est alors posée aux groupes qui doit leur servir de ligne directrice de lecture: «À quels changements qui s'opèrent à l'époque de François Rabelais les récits font-ils écho?» Pour illustrer notre propos, nous nous appuierons sur le travail produit par le groupe d'étudiants en charge du thème « Apprendre ».

\section{Groupe : Apprendre}

Texte 1 -

“On fit apprendre [à Gargantua] l'alphabet si bien qu'il le disait par coeur à l'endroit et à l'envers durant cinq ans et six mois. Puis on lui lut Donat, le Facet, Theodolet et Alanus in Parabolis pendant treize ans et six mois et deux semaines.

Mais notez qu'on lui apprenait également à écrire gothiquement et qu'il écrivait tous ses livres, car l'art d'impression n'était encore en usage. [...]

Puis on lui lut De modis significandi, avec les commentaires de Hurtebize, de Fasquin, 
de Tropdireulx, de Gualehaul, de Jean le Veau, de Billonio, Brelinguandus et un tas d'autres, cela prit dix-huit ans et onze mois."

Gargantua, chapitre XIV

Texte 2 -

"J'entends et veux que tu apprennes les langues parfaitement : premièrement le grec, comme le veut Quintilien; deuxièmement, le latin; et puis l'hébreu pour les Saintes Lettres, et le chaldéen et l'arabe pareillement; et que tu formes ton style quant au grec, à l'imitation de Platon ; quant au latin de Cicéron. [...]

Des arts libéraux, géométrie, arithmétique et musique, je t'en ai donné le goût quand tu étais encore petit; laisse-moi l'astrologie divinatrice [...]. Du droit civil, je veux que tu saches les beaux textes et me les confère avec philosophie.

Et quant à la connaissance de la nature, je veux que tu t'y adonnes curieusement : qu'il n'y ait mer, rivière, ni fontaine dont tu ne connaisses les poissons ; tous les oiseaux de l'air, tous les arbres, arbustes et fruits des forêts, toutes les herbes de la terre, tous les métaux cachés au ventre des abîmes [...]."

Pantagruel, chapitre VIII

Dans un deuxième temps, chaque groupe doit rédiger un court paragraphe reprennant les principaux éléments de réponse qui ont été apportés à la question posée. La consigne de rédaction impose aux étudiants de construire un récit: "Gargantua parle et présente à son fils Pantagruel les changements qui sont en train de s'opérer à son époque ». Ce deuxième temps doit permettre, par le récit, de créer une première étape de sens. Nous nous référons ici aux travaux de Nicole Lautier (Lautier, 2003) et de Didier Cariou (Cariou, 2012) sur l'écrit en histoire.

Le dernier temps de cet exercice est à proprement parler un exercice d'» hybridation/ fragmentation ", autre exercice emprunté à l'OuBaPo. L'objectif est ici, pour le groupe, de réaliser une planche de bande dessinée au format A4 qui conduira à produire un iconotexte en faisant dialoguer le texte produit à la phase précédente et une image sélectionnée qu'il conviendra de découper et de séquentialiser. Au groupe "Apprendre ", nous avons confié trois images du XVIe siècle : deux gravures d'Albrecht Dürer réalisées en 1513-1514, Melencolia et Saint-Jérôme dans sa cellule; une estampe de Philippe Galle réalisée vers 1620-1630, Cosmographe dans son cabinet de travail.

Ce troisième exercice demande aux étudiants de passer d'une production linéaire et textuelle à une production tabulaire et iconotextuelle. Son intérêt réside donc dans le passage d'une production à l'autre et dans l'adaptation des étudiants, par ce que Muniesa et Callon nomment la "performation ", au langage de la bande dessinée. Pour ces deux sociologues, la " performation » est le fait qu'un ensemble d'activités et d'événements instaurent ou modifient un agencement préexistant (Muniesca, Callon, 2013), instaurent ou modifient une pensée sur un concept.

Le groupe «Apprendre » du troisième exercice, si nous poursuivons avec notre exemple, a choisi de réaliser un texte qui prend la forme d'une lettre de Pantagruel adressée à son père, Gargantua :

Cher père,

Sachez qu'apprendre ne correspond plus aux us de notre temps. Voyez-vous, vous qui passiez (des heures) [votre temps] à apprendre par cœur l'alphabet, c'est au fil de mes lectures que j'ai connu les revers de la langue et sa calligraphie pendant que vous passiez votre temps à lire et relire, écrire et réécrire les mêmes ouvrages. 
Désormais, [Grâce aux livres,] j'apprends à connaître le monde qui m'entoure en m'intéressant aux arts libéraux, [les arts], l'astronomie et à la nature [les mathématiques]. Du grec, du latin, de l'hébreu, du chaldéen et de l'arabe, j'ai fait mes plus fidèles amies. Elles m'ont permis de m'ouvrir à de nouveaux savoirs qui font de moi l'être instruit que je suis. La nature n'a plus de secret pour moi, elle regorge de trésors que vous n'avez pas eu la chance d'approcher... Vous qui consacriez votre temps à un apprentissage vide de sens mais auquel on reconnaît une certaine rigueur. Les sages m'ont initié à la philosophie, j'ai grandi. [... et mes pensées s'envolent vers vous : elles partent en voyage !]

Bien à vous, de gargantuesques baisers et pensées,

Votre fils bien aimé,

Pantagruel

Si l'on se réfère aux deux textes proposés au groupe ( $c f$. partie 2), nous constatons que les éléments du passé (texte tiré de Gargantua) et les éléments du présent (texte tiré de Pantagruel) sont mis en opposition de manière constante dans le texte. De plus, la contrainte de la mise en récit a développé la créativité du groupe qui fait le choix du récit épistolaire et d'un style inspiré de la langue du XVI ${ }^{\mathrm{e}}$ siècle.

Le groupe "Apprendre » a ensuite choisi de découper l'estampe de Philippe Galle qui lui a paru plus riche en détails. Avant de se lancer dans le découpage et dans le collage sur la planche $\mathrm{A} 4$, le groupe a réalisé un brouillon que l'on peut associer à un story-board dans lequel ne figure aucun crayonné mais des indications spatiotopiques et séquentielles (présence de flèches soulignant l'enchaînement logique des cases, par exemple). Les éléments textuels présents sur le brouillon sont à la fois des indications iconiques (sablier, livres, globe, bateau...) et des indications textuelles (les arts, l'astronomie, « votre fils bien aimé »). Le brouillon comporte cinq strips dont le quatrième est vide et très large comme si les idées s'étaient taries pour rejoindre la case finale qui, elle, est déjà en place et reprend la signature de la lettre rédigée lors de la phase 2 . 
Image 5 - Brouillon du groupe « Apprendre»

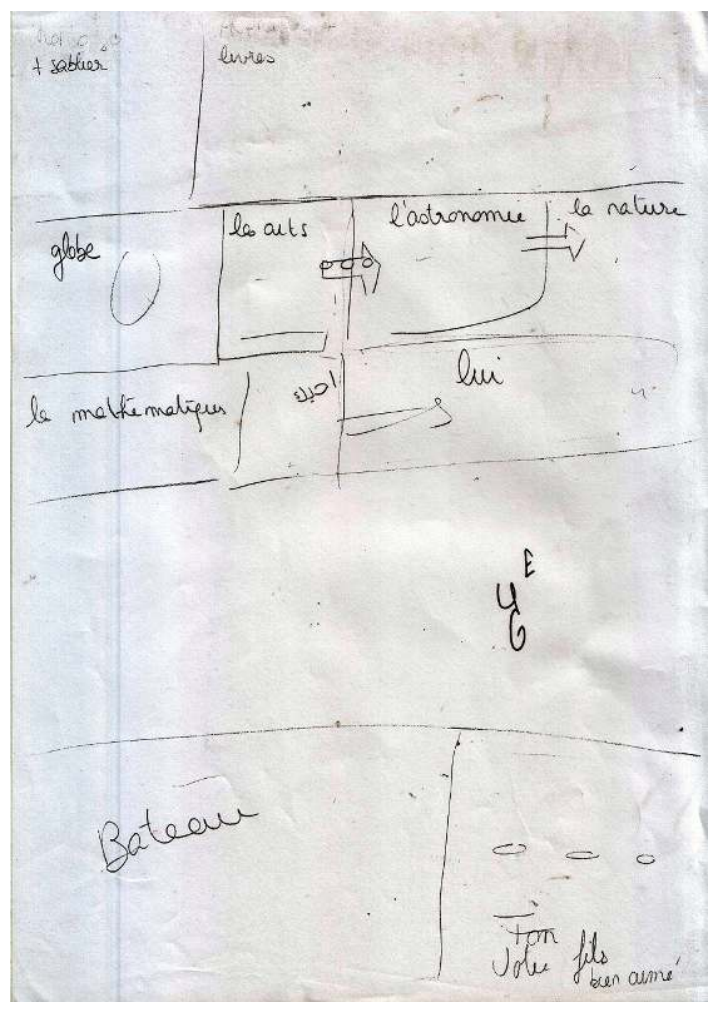

Image 6 - Planche du groupe « Apprendre »

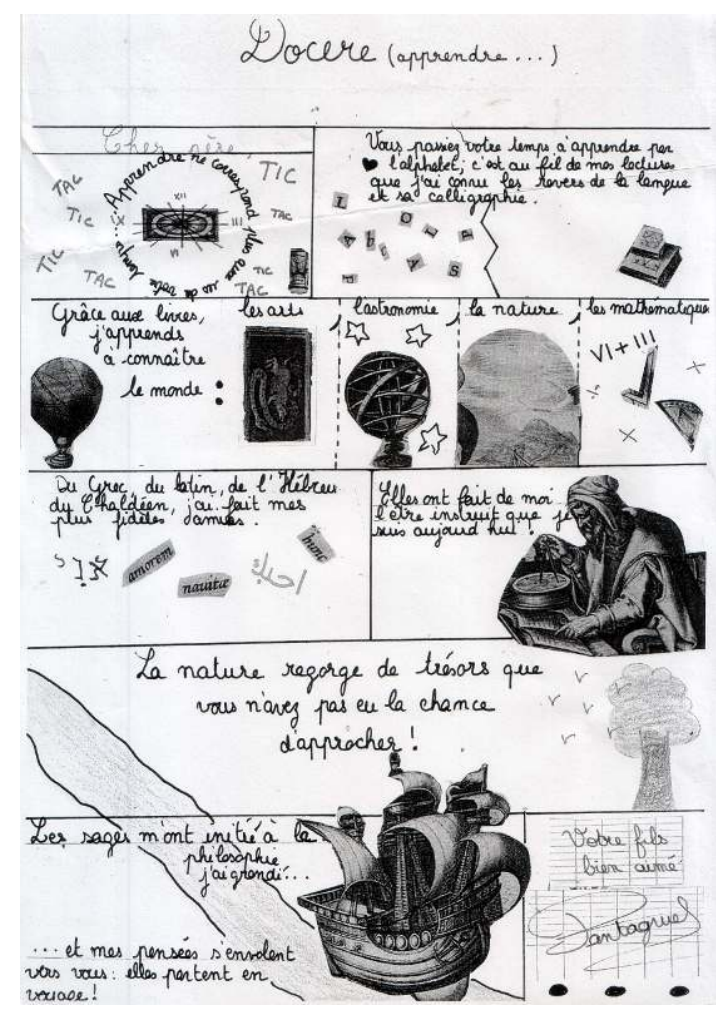

La formule latine utilisée en titre replace le thème dans son contexte thématique. La planche comporte au final cinq strips de même largeur. La lettre, produite en phase 2, a 
été totalement redécoupée mais conserve toutefois sa forme épistolaire (adresse, formule d'envoi) :

Cher père,

vous passiez [votre temps] à apprendre par cœur l'alphabet, c'est au fil de mes lectures que j'ai connu les revers de la langue et sa calligraphie

[Grâce aux livres,] j'apprends à connaître

[les arts], l'astronomie la nature [les mathématiques]. Du grec, du latin, de l'hébreu, du chaldéen et de l'arabe, j'ai fait mes plus fidèles amies. Elles font de moi l'être instruit que je suis. La nature, regorge de trésors que vous n'avez pas eu la chance d'approcher...

Les sages m'ont initié à la philosophie, j'ai grandi. [... et mes pensées s'envolent vers vous : elles partent en voyage !]

Votre fils bien aimé, Pantagruel

41 Le premier strip situe l'action et le sujet: il s'agit d'une lettre d'un père à son fils qui cherche à opposer deux générations par leur rapport au savoir. La première case accumule les références au temps qui passe (cadran solaire, sablier, tic-tac d'une horloge). La case suivante oppose des lettres dissociées de l'alphabet à des livres dans lesquels les lettres ont été agencées pour faire sens. Le strip suivant comporte quatre cases séparées par des tirets. Dans une progression thématique à sous-thèmes dérivés, les quatre cases du strip suivant illustrent les différents savoirs rendus possibles et accessibles par le livre. Les images juxtaposées, tirées de l'estampe, coïncident avec l'énumération du texte. Le rythme ralentit par le passage à deux cases longues du strip 3 qui se clôt sur l'image du cosmographe de Galle. Ce dernier symbolise « l'être instruit » qu'est devenu Pantagruel.

Les deux derniers strips sont réunis par un cours d'eau qui porte le bateau, métaphore de la découverte du monde par le voyage. Le texte et les images font référence à un même champ lexical qui évoque l'ouverture et la libre pensée : le cours d'eau, le bateau, la philosophie, l'envol de la pensée, le voyage. On se rend compte ainsi que l'iconotexte produit par le groupe "Apprendre ", par l'interaction entre le récit textuel et le récit iconique (Boulaire, 2006), permet aux étudiants de créer davantage d'effets de sens, de complexifier le récit en jouant avec les effets du multicadre.

L'exercice proposé permet aux étudiants de s'approprier des connaissances historiques ou tout au moins de les consolider. L'interdépendance texte/image peut constituer un mode d'élaboration de la pensée qui semblerait intéressant de mettre en œuvre en classe avec des élèves du primaire ou du secondaire. Si les étudiants ne l'expriment pas en ces termes, ils manifestent dans leurs impressions post-action que l'exercice leur a permis d'apprécier que le langage de la bande dessinée pouvait être "utile et efficace ». Tous les étudiants ont apprécié ce dernier exercice qui leur a montré une autre manière de «travailler sur la BD». Certains affirment avoir découvert « que la BD pouvait servir de support et non seulement de lecture récréative ».

Globalement, la demi-journée baptisée «La BD s'invite à l'ESPE » est plutôt un succès. Ce temps a permis aux étudiants de lever le voile sur un médium qui leur était finalement très méconnu voire inconnu. Les ateliers qui ont suivi ont été bien accueillis dans l'ensemble. La très grande majorité a salué les côtés pratiques, didactiques et pédagogiques. Les activités proposées dans les ateliers ont permis pour beaucoup de voir ce qu'il était possible de faire avec des enfants d'âges très différents et de préciser les 
usages didactiques qui pouvaient être mis en place à partir du médium pour en expliquer le fonctionnement systémique ou bien à partir du langage de la bande dessinée pour construire des savoirs disciplinaires. Ces ateliers, qu'ils ont jugés « ludiques », sont une plus-value par rapport à ce que les étudiants ont l'habitude de faire à l'ESPE.

«La BD s'invite à l'ESPE » est encore une action nouvelle en cours de stabilisation. Les étudiants qui ont participé aux deux sessions n'ont pas manqué de suggérer des pistes pour améliorer encore son organisation et son contenu montrant leur intérêt pour ce qu'ils considèrent comme un vrai temps de formation. Ainsi proposent-ils la réalisation d'une exposition de leurs travaux afin de revenir sur la transposition de ces exercices dans les classes et de valoriser ce temps-fort auprès des étudiants de seconde année de master, déjà en poste et qui n'ont pas participé à l'action. Depuis 2016, certains de ces ateliers destinés aux étudiants de première année de master MEEF ont donné matière à un certain nombre de mémoires professionnels en seconde année portant, par exemple, sur les combats de la Première guerre mondiale dans la BD, ou l'enseignement de la géographie en cycle 3 par la BD. Certains étudiants se sont interrogés sur la pertinence d'avoir recours à la BD pour enseigner la Shoah à l'école primaire. Dans la grande majorité de ces travaux, les étudiants-stagiaires font réaliser à leurs élèves une planche ou passent par des exercices oubapiens et reconnaissent que ces derniers permettent aux élèves de s'emparer d'un médium avec lequel ils ne semblent pas, ou sans doute plus, aussi familiers.

\section{Conclusion}

Dans des maquettes de formation toujours plus contraintes, trouver du temps pour initier les futurs professeurs d'école aux richesses des supports littéraires qu'ils seront amenés à proposer à leurs élèves relève de la gageure. Toutefois, créer un événement autour de la bande dessinée peut leur faire découvrir, leur donner à penser et leur permettre d'envisager des modalités de traitement en classe de la bande dessinée variées et attentives à la richesse du médium. Initier à la didactique d'un texte littéraire spécifique et à la manipulation du langage narratif de la bande dessinée par une pratique personnelle transférable semble porter ses fruits même sur un temps limité, ainsi que le montre un sondage réalisé a posteriori.

47 L'ensemble des activités proposées dans les divers ateliers de ce temps-fort consacré à la $\mathrm{BD}$ à l'école contribue à faire prendre conscience aux étudiants-stagiaires que la bande dessinée est, comme Thierry Groensteen le soutient, un langage, c'est-à-dire "un ensemble original de mécanismes producteurs de sens » (Groensteen, 1999), dépassant la conception qui n'en ferait qu'un simple lieu de rencontre entre le texte et l'image. Langage à dominante visuel, la bande dessinée ne peut être analysée en séparant la narration iconique de la narration textuelle. Les ateliers présentés ont permis aux étudiants de s'intéresser aux articulations, à la distribution des espaces à travers la page ou, la double-page. Système sémiotique s'appuyant sur deux matières d'expression et la combinaison de codes, la bande dessinée constitue également un mode d'expression permettant d'écrire pour construire des savoirs. Si, comme l'a montré Vygotsky, en se transformant en langage, la pensée se réorganise et se modifie (Vygotsky, 1997). Cette même pensée, qui participe aux apprentissages, se réalise dans l'iconotexte. Élaborer une bande dessinée pour construire un récit sur un processus ou un phénomène historique comme le changement de paradigme entre l'éducation médiévale et l'éducation moderne, 
par exemple, passe par un cheminement depuis le «brouillon " vers la "mise au noir » qui relève de l'activité complexe participant de la compréhension du phénomène et de la construction de savoirs. Le recours à l'iconotexte, et plus particulièrement à la bande dessinée, conduit les élèves à mettre en interdépendance deux matières d'expression : activité exigeante et porteuse d'une diversité d'apprentissages, dont les conditions de mise en œuvre aux différents niveaux restent à expérimenter et à déterminer.

\section{BIBLIOGRAPHIE}

Allen, K., \& Ingulsrud, J. E. (2007). Strategies used by children when reading manga. Paper presented at the Annual Congress of the Applied Linguistics Association of Australia, New South Wales, Australia.

Callon, M. \& Muniesa F. (2013). La performativité des sciences économiques, In Ph. Steiner, F. Vatin, Traité de sociologie économique, (pp.281-313). Paris : PUF.

Cariou, D. (2012). Écrire l'histoire scolaire. Quand les élèves écrivent en classe pour apprendre l'histoire. Rennes : Presses universitaires de Rennes, coll. « Paideia ».

Dardaillon, S. (2009). Quelle place pour l'iconotexte dans les pratiques des enseignants du cycle 3 ? In B. Louichon et A. Rouxel (Dir..), La Littérature en corpus (pp. 85-95). Dijon : Scéren-CRDP Bourgogne.

Dardaillon, S. \& Meunier, C. (2012). Étudier une œuvre intégrale en bande dessinée au cycle 3 : quelles spécificités didactiques? In N. Rouvière (Dir.), Bande dessinée et enseignement des humanités, (pp. xx-xx). Grenoble : ELLUG

Franquin, Jijé (1969). Comment on devient créateur de bandes dessinées. Bruxelles : Dupuis.

Groensteen, T. (1999). Système de la bande dessinée. Paris : PUF.

Lautier N. (2003). Histoire enseignée, histoire appropriée. Quelques éléments spécifiques d'une didactique de l'histoire. In M.-C. Baquès, A. Bruter \& N. Tutiaux-Guillon (Dir.), Pistes didactiques et chemins d'historiens, textes offerts à Henri Moniot. (pp. 357-380). Paris : L'Harmattan.

Masson, P. (1985). Lire la bande dessinée. Lyon : Presses Universitaires de Lyon.

Nakazawa, J. (2004). Manga (comic) literacy skills as determinant factors of manga story comprehension. In Manga Studies, 5, (pp. 7-25).

Humoresques $\mathrm{n}^{\circ} 10$, L'humour graphique fin de siècle, Presses Universitaires de Vincennes et Centre National de la Bande Dessinée et de l'Image, 126 pages. Numéro réalisé par Thierry Groensteen. 2008

Vygotsky, L. (1997). Pensée et langage, Paris, La Dispute. 


\section{NOTES}

1. L'iconotexte est un terme créé au début des années 1980 par Michael Nerlich en France (par Kristen Alberg dans les pays anglosaxons) pour parler des productions culturelles dans lesquelles texte(s) et image(s) sont en interdépendance. La bande dessinée comme l'album pour enfants sont des iconotextes.

2. L'exercice d'hybridation consiste à croiser des images empruntées à une histoire avec un texte tiré d'une autre histoire. L'exercice de fragmentation consiste, quant à lui, à découper une image pour construire un récit séquentialisé. L'exercice proposé ici aux étudiants est la conjugaison de ces deux exercices oubapiens (cf. chapitre 3).

3. Mongo Sise, "Matamata et Pilipili”, Journal de Spirou, n²314, 19 août 1982, p.10-11.

\section{RÉSUMÉS}

Le présent article s'appuie sur une enquête et une expérimentation menées auprès d'étudiants de master Métiers de l'Enseignement et de la Formation premier degré. Il met en évidence la méconnaissance par la plupart d'entre eux du médium et analyse différents exercices didactiques mis en place dans le cadre d'une formation consacrée à la BD en classe. Ces exercices permettent de comprendre le fonctionnement de la $\mathrm{BD}$ et d'en utiliser le langage pour s'approprier des connaissances dans différents domaines disciplinaires, par exemple en histoire.

This article is based on a survey and an experiment conducted with master degree students in education. It highlights the lack of knowledge by most of them about the medium and analyzes various didactic exercises settled as part of a training devoted to comics in class. These exercises allow to understand how comics work and how the language of comics can be used to conceptualize in history, for example.

\section{INDEX}

Mots-clés : formation des enseignants, premier degré, bande dessinée, OuBaPo

Keywords : teachers training, Primary school, Comics, OuBaPo

\section{AUTEURS}

\section{SYLVIE DARDAILLON}

ESPE Centre Val de Loire, Université d'Orléans

CHRISTOPHE MEUNIER

ESPE Centre Val de Loire, Université d'Orléans 